\title{
Extended visual range: an observation during a total solar eclipse
}

\section{Michael Vollmer, Joseph Shaw}

Michael Vollmer, Joseph A. Shaw, "Extended visual range: an observation during a total solar eclipse," Proc. SPIE 11143, Fifteenth Conference on Education and Training in Optics and Photonics: ETOP 2019, 111431Q (2 July 2019); doi: 10.1117/12.2523167

SPIE Event: Fifteenth Conference on Education and Training in Optics and Photonics: ETOP 2019, 2019, Quebec City, Quebec, Canada 


\title{
Extended visual range: an observation during a total solar eclipse
}

\author{
Michael Vollmer ${ }^{1 *}$, Joseph A. Shaw ${ }^{2}$ \\ 1 University of Applied Sciences Brandenburg, Magdeburgerstr. 50, 14770 Brandenburg, Germany \\ 2 Montana State University, Bozeman, Montana 59717, USA
}

\begin{abstract}
Solar eclipses are magnificent natural phenomena during which the sun is obscured by the moon. Besides the unique opportunity of studying the solar corona and immediate vicinity of the sun, an eclipse also leads to a darkened daytime sky with sunset colors and many other fascinating phenomena. Here we focus on how the daytime horizontal visual range changed during the duration of the solar eclipse of 21 August 2017, observed from Rexburg, Idaho, USA. Close to totality the eastern horizon for a short time period showed the contours of the Grand Teton Mountains from distances between about $80 \mathrm{~km}$ to $90 \mathrm{~km}$. We show and discuss photographic images that show the visual range during totality being significantly extended beyond the visual range in most of the partial phase before and after totality, which was below $80 \mathrm{~km}$ when the mountains could not be seen by the naked eye. This phenomenon of an extended visual range can be explained in terms of a simple model for the daytime visual range. This model, which will be explained in this presentation, nicely reproduces the observations and also predicts other phenomena; for example, it predicts that similar phenomena may be observed if part of the line of sight close to the observer is in deep shade of a thick cloud cover. The presentation will tie these observations and their explanation to the teaching of optical scattering and atmospheric optics.
\end{abstract}

Keywords: Optics education, visual range, solar eclipse

\section{INTRODUCTION}

Daylight visual range [1-5] describes the maximum distance at which an observer is able to detect objects such as mountain peaks versus a clear sky background. Looking at distant objects, one always detects scattered light from the object, as well as air light, i.e. scattered radiation from the molecules and aerosols along the line of sight. The horizontal visual range depends on the contrast, which is related to the luminance difference between the object and the adjacent horizon sky (which only consists of air light). The luminance integrals usually are approximated by monochromatic averaged values for wavelength of $560 \mathrm{~nm}$. In this case, contrast $C$ is simply related to the scattering coefficient $\beta$ along a line of $\operatorname{sight}$ of length $x$ by $-\ln (C)=\beta$ $x$. Usually, one is just able to detect a contrast difference of $2 \%$, hence visual range $x=d$ (for range $d$ ) is defined by $\mathrm{C}=0.02$. One usually only needs to know the scattering coefficient $\beta$ to estimate the range $d$. For molecular scattering, one may use tables for $\beta$ (e.g. [6]) or an approximate formula [3]. For $\beta=0.0114 \mathrm{~km}^{-1}$ we find a visual range of about $340 \mathrm{~km}$, in close agreement to the value of $330 \mathrm{~km}$ based on numerical evaluations of the integrals [3]. However, this extremely large value is usually not observable in nature because any haze layer leads to larger $\beta$ values and reduces the range. In modern usage, values above $50 \mathrm{~km}$ are considered to reflect excellent visibility and more than $100 \mathrm{~km}$ visual range is exceptional.

Consider a usual summer day. There may be a little haze in the atmosphere, but as long as the scattering coefficient $\beta$ remains the same, a given visual range $d$ should not change. Knowing this, we were quite astonished when during totality of the 2017 solar eclipse, we suddenly could observe mountain tops well beyond the visual range of that day, before and after totality.

*vollmer@th-brandenburg.de; phone+493381355347

Fifteenth Conference on Education and Training in Optics and Photonics: ETOP 2019, edited by

Anne-Sophie Poulin-Girard, Joseph A. Shaw, Proc. of SPIE Vol. 11143, 111431Q · ( 2019

SPIE, ICO, IEEE, OSA · CCC code: 0277-786X/19/\$18 · doi: 10.1117/12.2523167

Proc. of SPIE Vol. $11143111431 \mathrm{Q}-1$ 
Not being the main topic today, the physics of eclipses is assumed to be well-known (see e.g. [7,8] and refs. therein). The only fact we need to consider is that the direct sun is shadowed by the moon, i.e. the available sunlight irradiating the atmosphere and thus responsible for the air light - relevant for the visual range definition - is changing during the eclipse. This provides an excellent natural optics example that can be used to teach about the effects of optical scattering on visual scenes.

\section{THE ECLIPSE}

On 21 August 2017, a total solar eclipse passed through the northwestern U.S. state of Idaho while crossing through the continental United States. We were making observations at a small astronomical observatory near Rexburg, Idaho $\left(43.8293^{\circ} \mathrm{N}, 111.8851^{\circ} \mathrm{W}\right)$. The site was close to the center line, resulting in us being in totality for about $2 \mathrm{~m} 17 \mathrm{~s}$. The remote observatory location, beyond the edge of town and some $300 \mathrm{~m}$ from the nearest road, resulted in negligible light pollution. Figs. 1 and 2 show example photos of the horizon sky with the camera pointed toward the Teton mountain range, located at a distance of about $80 \mathrm{~km}$ to $90 \mathrm{~km}$ east of the observatory. The center of the path of totality was just slightly south of the Grand Teton peak. As a simplification, we assume that both the observing location and the observed Teton peaks were close to the center line.
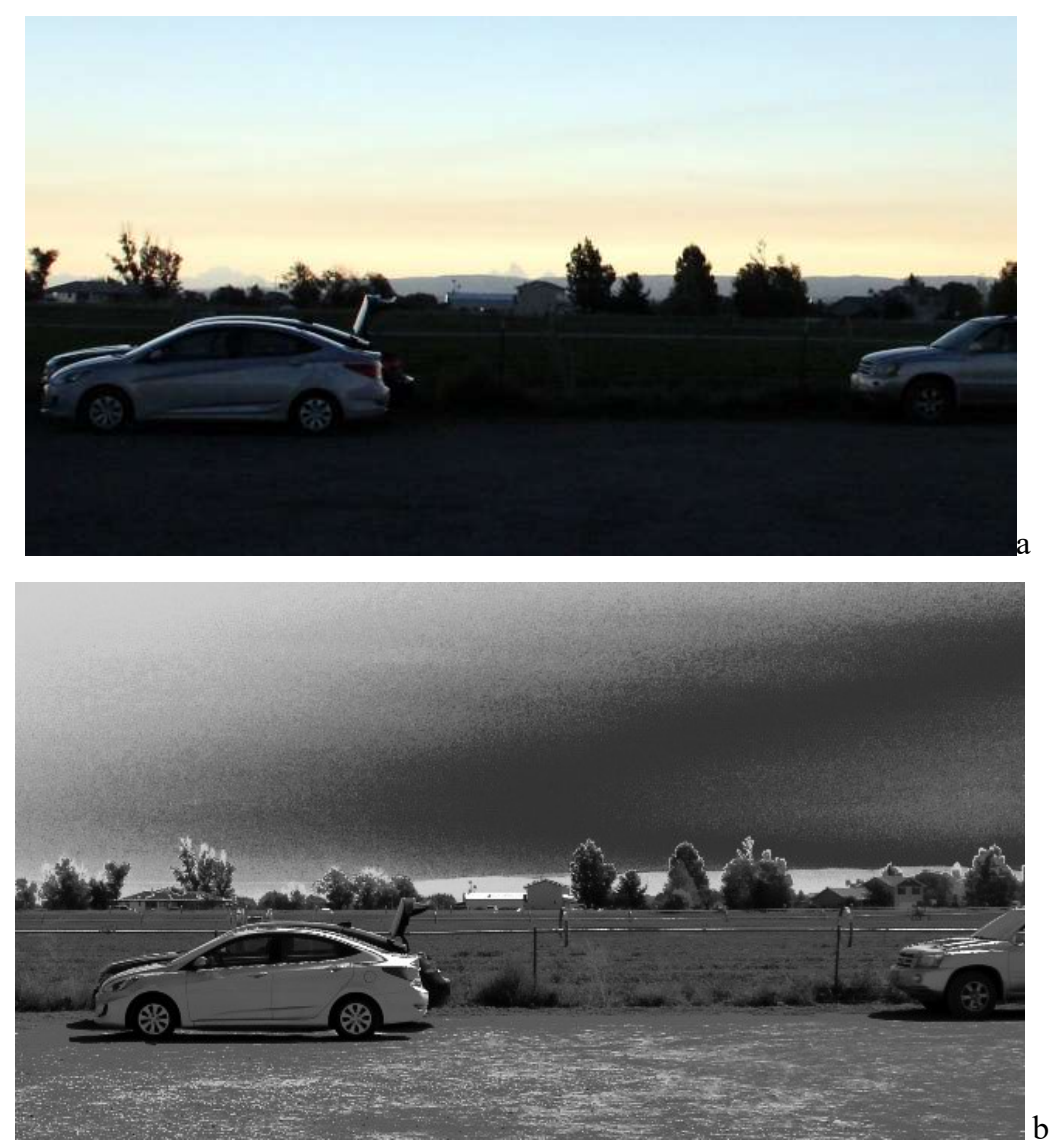

Figure 1. Photos during totality show mountain ridges (a) which are no longer visible 46 min later (b). 
Figure 1a shows a contrast-enhanced and cropped image recorded $36 \mathrm{~s}$ after the beginning of totality, in which the Teton peaks are visible just above the dark foreground. Figure $1 \mathrm{~b}$ shows the same scene about 46 minutes after totality. All imageprocessing attempts to make small contrast differences visible by changing to grey scale and nonlinearly modifying the gradation curve (Photoshop) in Fig. 1b were in vain: the mountain ranges could not be seen any more. Similarly, Fig. 2 shows the left-hand-side peaks of Fig. 1 enlarged with a telephoto lens after about $45 \mathrm{~s}$ of totality. This photo is original and not manipulated by image processing.

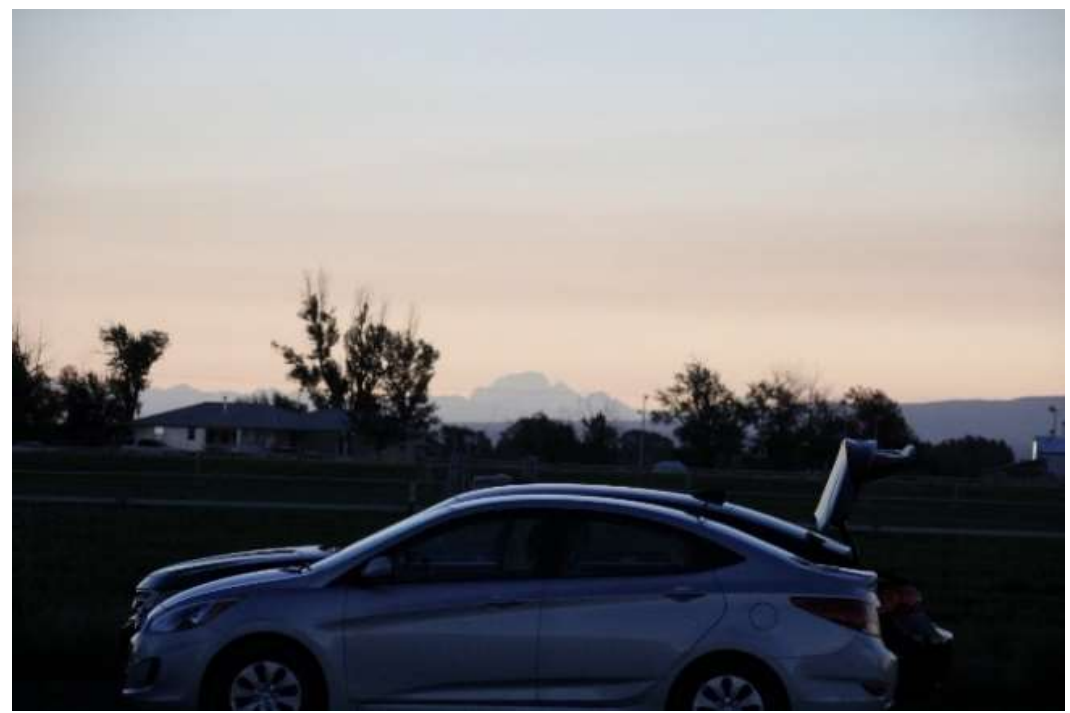

Figure 2. Photos recorded $45 \mathrm{~s}$ into totality showing a closer view of the left-hand-side mountain ridges of Fig. 1.

In order to correlate the observation with the Grand Teton mountains, we compared the images to the same scene photographed on a much clearer day from a slightly shorter distance and similar direction. This allowed unequivocal identification of the major peaks on the photo (Mt. Owen, Grand Teton and Middle Teton) with distances between $80 \mathrm{~km}$ and $90 \mathrm{~km}$ from the observatory where we were during the eclipse.

To summarize the observations: before and after totality, the mountains were invisible, i.e. the visual range was below 80 $\mathrm{km}$. However, during totality, the visual range must have increased well above $90 \mathrm{~km}$ such that the distant peaks became visible. It is this phenomenon which was investigated in detail.

\section{SIMPLIFIED MODEL FOR VISUAL RANGES}

The typical simplified model for daylight visual range according to Bohren and Fraser $[3,4]$ was already summarized above. It depends on the contrast, which is related to the luminance difference between the object and the adjacent horizon sky, the latter being due to air light. All applied simplifications in the approach were discussed in [5].

The main problem consists of calculating contrast from the radiances of an object and the horizon, which contrast is altered by the air light contributions. For example, for incident solar radiance $L_{0}$ and horizon sky radiance $L_{h o}$, the radiance $L_{o b j}$ of a dark object viewed from distance $d$ along a line-of-sight path homogeneously irradiated by sun light is given by 


$$
L_{o b j}=L_{0} G\left(1-e^{-\beta d}\right) \quad \text { and } \quad L_{h o r}=L_{0} G
$$

where $\mathrm{G} \approx 10^{-5}$ accounts for the geometry associated with isotropic molecular scattering [5]. Using these radiances and monochromatic luminance estimates, the visual range condition for contrast $C=0.02(-\ln C=3.9)$ yields the result $d \approx 3.9 / \beta \approx$ $340 \mathrm{~km}$ for molecular scattering $\left(\beta=0.0114 \mathrm{~km}^{-1}\right)$ on a hypothetical day with a purely molecular atmosphere.

For the actual hazy eclipse day (the haze was from wildfire smoke and dust from agricultural harvesting), we estimated the aerosol scattering coefficient to be approximately $\beta_{\text {aer }}=0.05 \mathrm{~km}^{-1}$, which, together with molecular scattering, yielded a total scattering coefficient, $\beta_{\mathrm{tot}}=0.064 \mathrm{~km}^{-1}$, and a reduced visual range of approximately $63.5 \mathrm{~km}$ [5]. This is in accordance with the observation that the Teton peaks could not be seen with the naked eye from Rexburg prior to totality.

We next extend this discussion to consider the situation where inhomogeneous irradiation of the atmospheric path leads to a change in the relative object and horizon sky radiance, which in turn produces a change in contrast (and hence visual range).

For our discussion, the only major parameter that changes during an eclipse is that the atmospheric path along the line of sight will be irradiated inhomogeneously. The top image in Fig. 3 depicts the general geometry and the bottom image in Fig. 3 schematically illustrates how the incident radiance varies during an eclipse.
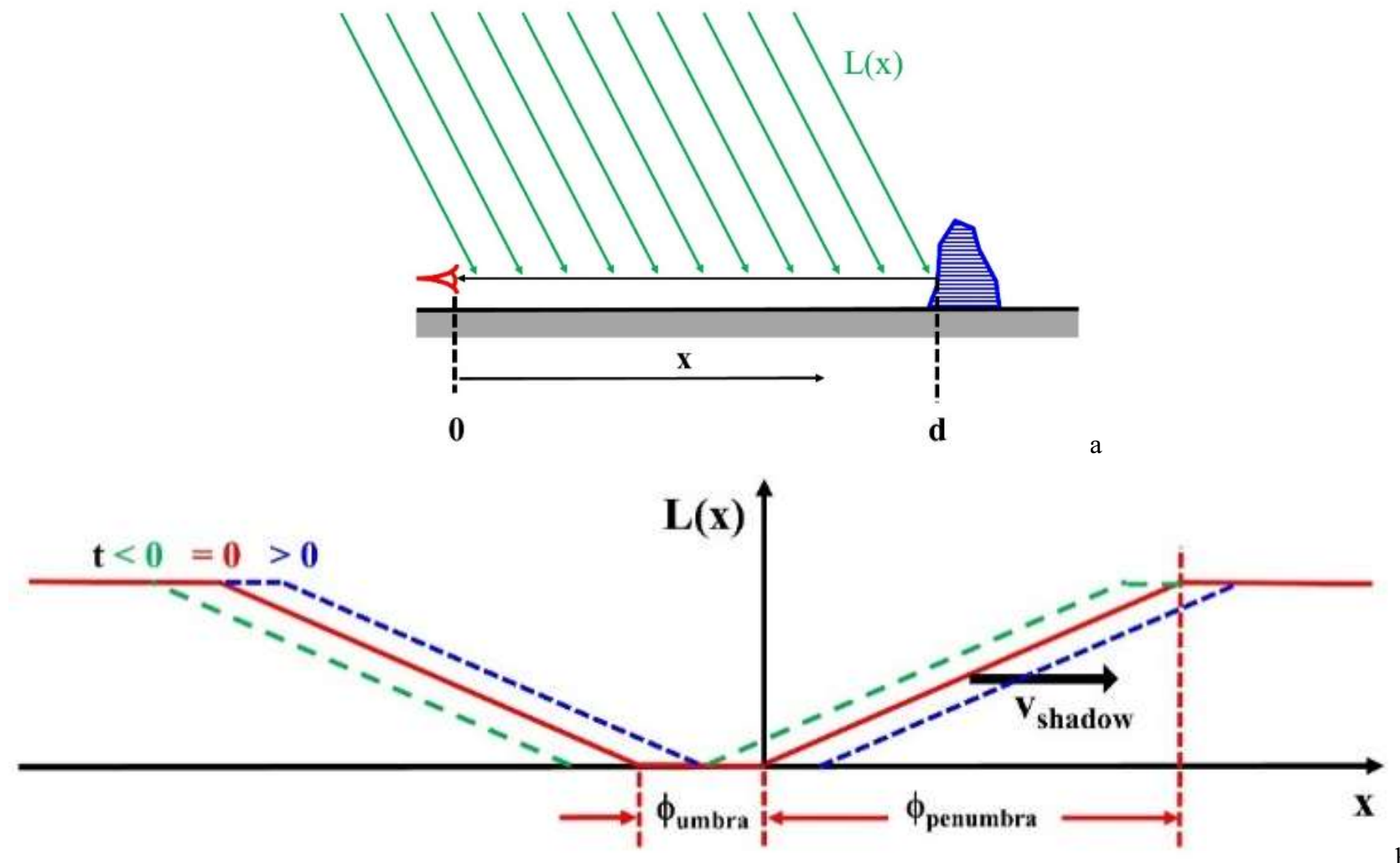

Fig. 3: a) schematic overview; b) observation situation with radiance variation along the line of sight during an eclipse. Scales are distorted: typical penumbra radius $\geq 3000 \mathrm{~km}$, typical umbra diameter around $100 \mathrm{~km}$ 
The chosen linear gradient is a rough, though astonishingly accurate, approximation for most of the partial phase of the eclipse [9]. In order to compute radiance as a function of time, we used the known moon's shadow velocity during the 2017 eclipse in Idaho of about $v=839 \mathrm{~m} / \mathrm{s}$. We separately calculated the object radiance along the line of sight, as well as the horizon sky radiance, along a path from observer to infinity [5].

\section{RESULTS AND DISCUSSION}

Figure 4 shows the final result for the contrast evaluated with this model for the observer position looking towards the Grand Teton Mountains as a function of time for two different scattering coefficients in an overview (Fig.4a) and with an expanded scale around the threshold of $C=2 \%$ (Fig. $4 \mathrm{~b}$ ).
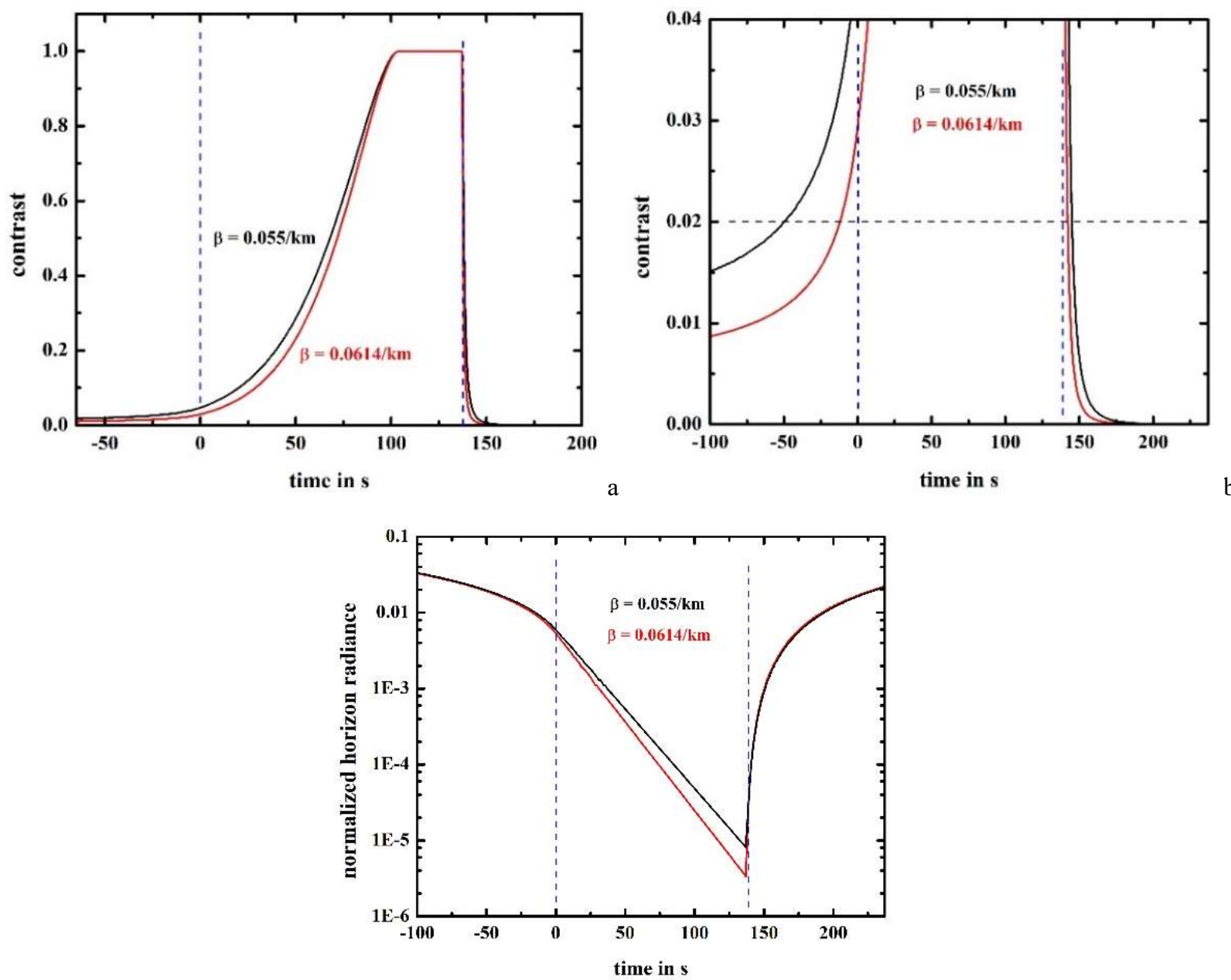

Figure 4: Model contrast and horizon brightness as a function of time before and after the eclipse for an object distance of $87.5 \mathrm{~km}$. a) contrast overview; b) closer view of the contrast in the vicinity of $C_{\text {thresh }}=0.02$ (horizontal broken line); c) normalized horizon brightness for two different scattering coefficients. Start and end of totality at observer position were at $t=0$ and $t=137 \mathrm{~s}$, indicated by blue vertical broken lines. 
The lower curves correspond to the previously discussed typical value of $\beta=0.0614 \mathrm{~km}^{-1}$ for the estimated haze at the observation site and day of the eclipse, while the second one is for a slightly less hazy atmosphere to see the respective differences.

The contrast is clearly asymmetric, as expected. The same applies, however, to horizon sky brightness normalized to the value before and after the eclipse (Fig. 4c). Similar to illuminance measurements, it may drop by about four orders of magnitude within just minutes, but it is rather asymmetrical. The minimum light level during totality still allow color vision, i.e. even this low light level seems adequate for detecting contrast between distant objects and the horizon sky.

Fig. 4 b shows that, for the chosen scattering coefficients, the mountains will only become visible $(C>0.02)$ to the naked eye around $50 \mathrm{~s}$ or less prior to totality. The observation window will then close again around $10 \mathrm{~s}$ after totality, leaving a period of around 3 minutes and $20 \mathrm{~s}$ overall for observing the mountains with an extended visual range. This is truly a very rare phenomenon, probably even more rare than the window of totality itself, since you also need favorable visual range conditions and suitable objects.

\section{CONCLUSIONS}

We have shown evidence that during the total solar eclipse on 21 Aug. 2017, the greatly reduced scattered air light along the observation path from our observation site allowed the 80-90 km distant Grand Teton Mountains to become visible during totality and immediately prior to totality. We have presented a simple model for the horizontal visual range, which agrees with the observations. It would be interesting to conduct further experiments during future eclipses to enable further refinement and validation of the theory. Such experiments might be conducted, for example, by positioning a camera to view distant mountains that are not visible in regular daylight. The principles discussed here provide an interesting context for teaching about optical scattering and its impact on what we see in every-day (and not so every-day) scenes.

\section{Acknowledgments}

JAS acknowledges support from the U.S. Air Force Office of Scientific Research under agreement number FA955014-10140. This paper summarizes material published previously in [5].

\section{REFERENCES}

[1] Koschmieder, H, “Theorie der horizontalen Sichtweite,” Beiträge zur Physik der freien Atmosphäre XII, 33-53 (1924).

[2] Koschmieder, H, “Theorie der horizontalen Sichtweite II,” Beiträge zur Physik der freien Atmosphäre XII, 171-181 (1924).

[3] Bohren, C. F., Fraser, A., “At what altitude does the horizon cease to be visible?” Am. J. Phys. 54(3), 222-227 (1986).

[4] Bohren C. F., Clothiaux, E. E., [Fundamentals of atmospheric radiation], Wiley (2006).

[5] Vollmer, M., Shaw, J.A., "Extended visual range during solar eclipses", Appl. Opt. 57(14), 3250-3259 (2018)

[6] Penndorf, R., "Tables of the refractive index for standard air and the Rayleigh scattering coefficient for the spectral region between 0.2 and $20 \mu$ and their application to atmospheric optics," J. Opt. Soc. Am. 47(2), 176-182 (1957)

[7] Littmann, Willcox, M., K., [Totality, Eclipses of the sun], U Hawaii Press (Honolulu) (1991).

[8] https://eclipse2017.nasa.gov/sites/default/files/interactive map/index.html

[9] Möllmann K.-P., Vollmer, M., "Measurements and predictions of the illuminance during a solar eclipse," Eur. J. Phys. 27, 1299-1314 (2006) 